\title{
Persistent Current Fluctuations in Mesoscopic Rings in the Presence of Spin Effects
}

\author{
Ulrich Eckern* \\ Kernforschungszentrum Karlsruhe, Institut für Nukleare Festkörperphysik, Postfach 3640, W-7500 Karlsruhe 1, Germany
}

\begin{abstract}
The effects of spin-flip scattering, spin-orbit interaction, and Zeeman splitting on the persistent currents in mesoscopic metallic rings and related correlation functions are investigated, with emphasis on the non-interacting electron case. Interaction effects are briefly reviewed.
\end{abstract}

\section{Introduction}

Following the seminal paper of Büttiker, Imry and Landauer [1], the phenomenon of persistent currents in mesoscopic samples has been discussed theoretically in various papers [2-21]. Accordingly, a normal-metal ring threaded by a magnetic flux $\phi$ should carry an equilibrium current; i.e. a magnetic moment should appear as a consequence of the applied flux. Most important in the development of this field have been the experimental investigations of the two groups at Bell Labs [22] and at IBM [23], where flux dependent persistent currents have been shown to exist in mesoscopic metal rings.

The above references may serve as a guide to the more recent literature. In this article, I focus on how various spin effects modify the relevant correlation functions, aiming at a concise presentation. The following is based on standard perturbative methods, well known from the theory of "weak localization" [24-30], and thus subject to certain limitations $[14,19]$. Furthermore, I restrict myself to results related to the average current and its fluctuations (i.e. the two-point correlation function); arguments have been given $[17,18$, 21] which indicate that higher connected correlation functions are small, provided the total number of electrons in a ring is large.

The persistent current is an equilibrium phenomenon; hence the impurity average and the correlations of the thermodynamic potentials are central objects to be investigated, e.g.

$\mathscr{M}_{\Omega}=\left\langle\Omega(\mu, \phi) \Omega\left(\mu^{\prime}, \phi^{\prime}\right)\right\rangle_{c}$

Given $\mathscr{M}_{\Omega}$, one obtains easily by differentiation with respect to $\mu, \mu^{\prime}$ or $\phi, \phi^{\prime}$ the correlators of the particle number and the persistent current, respectively:

$\mathscr{M}_{N}=\left\langle\delta N(\mu, \phi) \delta N\left(\mu^{\prime}, \phi^{\prime}\right)\right\rangle_{c}$

and

$\mathscr{M}_{I}=\left\langle I(\mu, \phi) I\left(\mu^{\prime}, \phi^{\prime}\right)\right\rangle_{c}$

\footnotetext{
* Permanent address after 1 April 1993: Institut für Physik, Lehrstuhl für Theoretische Physik II, Universität Augsburg, Memminger Str. 6, W- 8900 Augsburg, Germany.
}

Being interested in the flux sensitive parts of these quantities, it is also possible, as becomes apparent from the structure of the results to be discussed below, to compute directly $\mathscr{M}_{N}$, integrate to obtain $\mathscr{M}_{\Omega}$, and differentiate with respect to the fluxes to obtain $\mathscr{M}_{I}$. On the other hand, a direct calculation of the current correlator is considerably more involved $[4,15]$.

An important step has been the understanding that the average persistent current has to be computed using the canonical potential, $F[5,6,9,11-13]$. The result is directly related to $\mathscr{M}_{N}$, namely

$\langle\delta F\rangle=\frac{1}{2} \Delta \mathscr{M}_{N}(\mu, \phi ; \mu, \phi)$

where $\Delta=\left(2 \mathscr{N}^{0} \mathscr{V}\right)^{-1}$ denotes the average level spacing $\left(\mathscr{N}^{0}\right.$ and $\mathscr{V}$ are the density of states at the Fermi surface for one spin and the volume).

On the other hand, interaction contributions $[7,8,11,17$, $21]$ are closely related to local fluctuations of the density,

$\mathscr{M}_{n}=\left\langle\delta n(\boldsymbol{r}, \mu, \phi) \delta n\left(\boldsymbol{r}^{\prime}, \mu^{\prime}, \phi^{\prime}\right)\right\rangle_{c}$

Interaction induced persistent currents are briefly discussed in Section 5.

\section{Non-interacting electrons - ideal case}

The diagrammatic representation of the relevant contributions to $\mathscr{M}_{\Omega}$ and $\mathscr{M}_{N}$ are shown in Figs 1 and 2 . In addition to the usual rules, note the combinatorial factor $M^{-1}$ in $\mathscr{M}_{\Omega}$, reflecting the symmetry of the diagram ( $M=5$ in Fig.

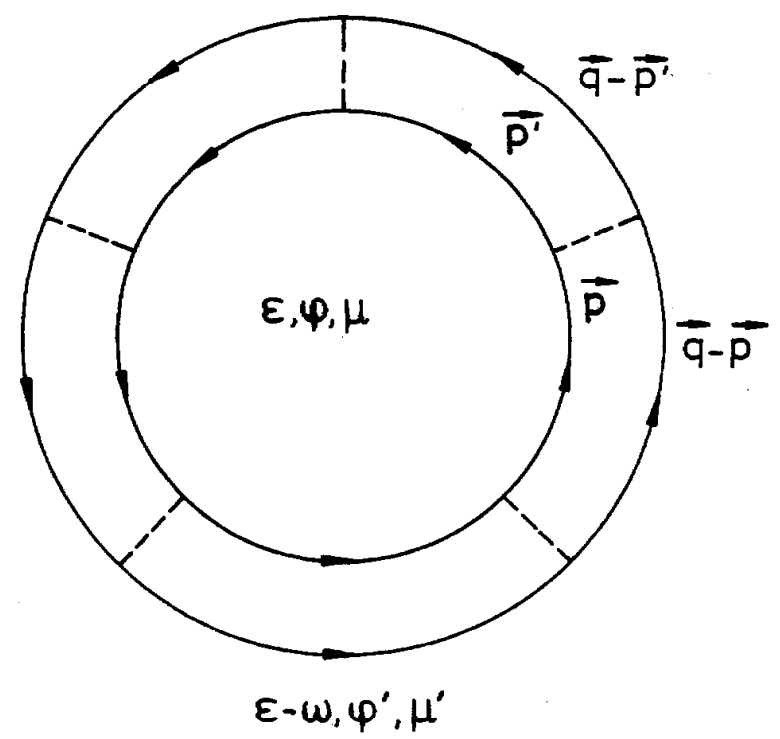

Fig. 1. Typical cooperon contribution to $\boldsymbol{\mu}_{\mathbf{\Omega}}$. For the corresponding diffuson, the arrows on one of the circles are reversed. The combinatorial factor is $\frac{1}{5}$, 


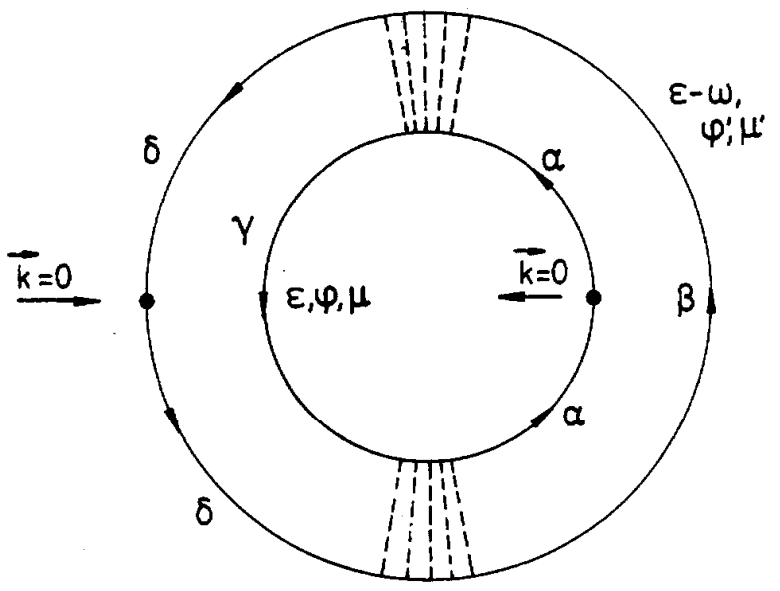

Fig. 2. Dominant cooperon contribution to $\mathscr{M}_{N}$

1). Flux sensitive contributions arise from the frequency regime $\varepsilon(\varepsilon-\omega)<0$; the result hence contains a factor $|\omega|$, and e.g. is $\left(\hbar=k_{B}=1\right)$

$\mathscr{M}_{\Omega}=-4 T \sum_{\omega, q} \frac{|\omega|}{2 \pi}\left[\ln \left(|\omega|+D q^{2}+i \delta \mu \operatorname{sgn} \omega\right)\right]_{C+D}$

Here $\delta \mu=\mu-\mu^{\prime}$, and the notation [... $]_{C+D}$ implies a sum over cooperon and diffuson contributions, i.e. for the ring geometry,

$q \rightarrow q_{ \pm}=\frac{2 \pi}{L}\left(n-\frac{\phi \pm \phi^{\prime}}{\phi_{0}}\right)$

respectively ( $\phi_{0}=h / e$ is the flux quantum, $L$ the perimeter). Clearly, (6) is of the form

$\mathscr{M}_{\Omega}=\mathscr{F}_{c}\left(\phi+\phi^{\prime}\right)+\mathscr{F}_{D}\left(\phi-\phi^{\prime}\right)$

where $\mathscr{F}_{C}$ and $\mathscr{F}_{D}$ are identical - and even - functions. This result, which follows from time reversal invariance, implies that the persistent current has the representation $[15,21]$

$I(\phi)=\sum_{m=1}^{\infty} I_{m} \sin \left(2 \pi m \phi / \phi_{0}\right)$

In order of magnitude (zero temperature), $\mathscr{M}_{\Omega} \sim E_{c}^{2}$, where $E_{c}=D / L^{2}$ is the Thouless energy, $\mathscr{M}_{N} \sim 1$, and $\mathscr{M}_{I} \sim$
$\left(E_{c} / \phi_{0}\right)^{2}$. More precisely,

$$
\left\langle I_{m}^{2}\right\rangle_{c}=96\left(e E_{c}\right)^{2} /\left(\pi^{2} m^{3}\right)
$$

Using (6) and (4), the average current is obtained $[11,13]$ as

$\left\langle I_{2 p+1}\right\rangle=0 ;\left\langle I_{2 p}\right\rangle=4 e \Delta / \pi^{2}$

i.e. its basic period is $\phi_{0} / 2$. Note that $\Delta \ll E_{c}$ for typical situations.

On the other hand, the local correlations,

$\mathscr{M}_{n}=\mathscr{V}^{-1} \int \frac{d^{3} k}{(2 \pi)^{3}}\left\langle\delta n_{k} \delta n_{-k}\right\rangle_{c} e^{i \mathbf{k}\left(r-r^{\prime}\right)}$

are given by the expression corresponding to Fig. 3, which has been discussed extensively in $[17,21]$ in connection with the phase sensitive impurity line. The result is

$$
\begin{aligned}
\left\langle\delta n_{\mathbf{k}} \delta n_{-\mathbf{k}}\right\rangle_{c}= & \frac{4 \operatorname{arctg} k l}{\pi k v_{F}} T \sum_{\omega, q} \\
& \times\left[\frac{|\omega|}{|\omega|+D q^{2}+i \delta \mu \operatorname{sgn} \omega}\right]_{C+D}
\end{aligned}
$$

which in order of magnitude leads to $\mathscr{M}_{n} \sim \mathscr{N}^{0} E_{c} / \mathscr{V}(l=$ $v_{F} \tau$ is the elastic mean free path), i.e. $\mathscr{M}_{n} / k_{F}^{6} \sim E_{c} /\left(N \varepsilon_{F}\right)$. Note that this result should be compared with the huge regular, flux insensitive contribution - given by a diagram similar to Fig. 3, except that only one impurity line connects the two rings - which is $\sim k_{F}^{5} / l$, i.e. the regular part is only by a factor $\sim\left(k_{F} l\right)^{-1}$ smaller than the square of the average density.

\section{Cooperon and diffuson in the presence of spin effects}

Here I collect the results for the spin dependence of the cooperon and the diffuson which, in one limit or the other, can be found at various places in the literature $[8,16,25-30]$. I assume that the spin-flip and the spin-orbit scattering rate ( $\tau_{s}^{-1}$ and $\tau_{s o}^{-1}$, respectively), as well as the Zeeman splitting, $\omega_{s}=2 \mu_{B} H$, are small compared to the non-magneticimpurity scattering rate, $\tau_{0}^{-1}$. At present, the total inverse mean free time is given by $\tau^{-1}=\tau_{0}^{-1}+\tau_{s}^{-1}+\tau_{s o}^{-1}$. The equations determining $C$ and $D$, in my notation [31]

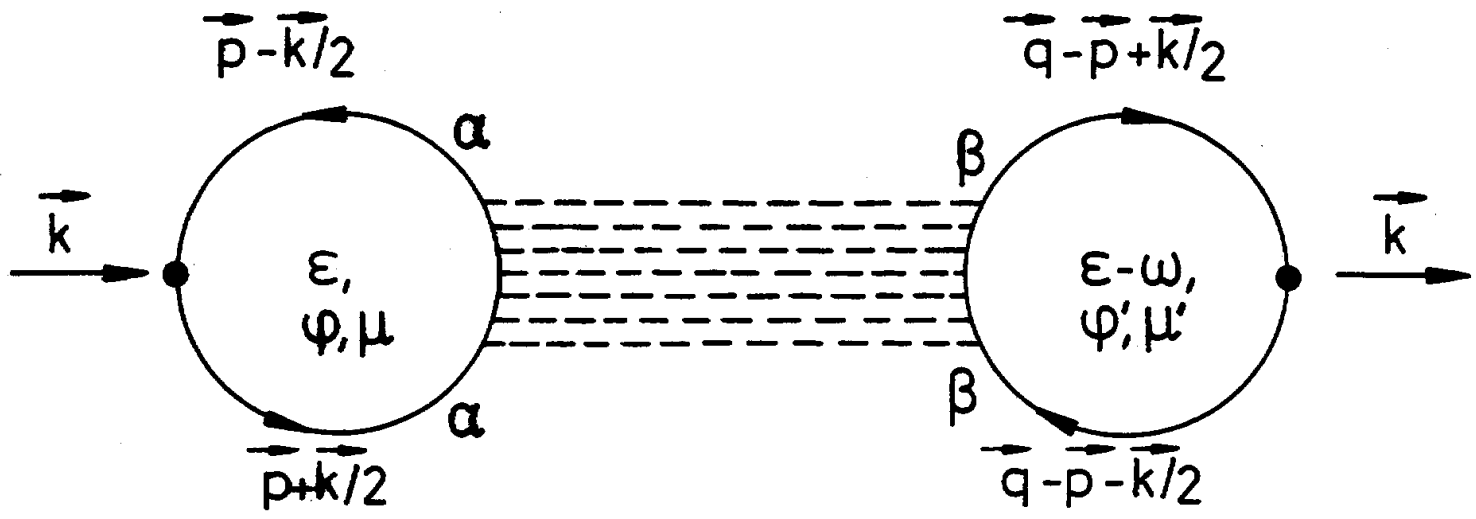

Fig. 3. Dominant cooperon contribution to the local correlator, $\mathscr{H}_{n}$. 


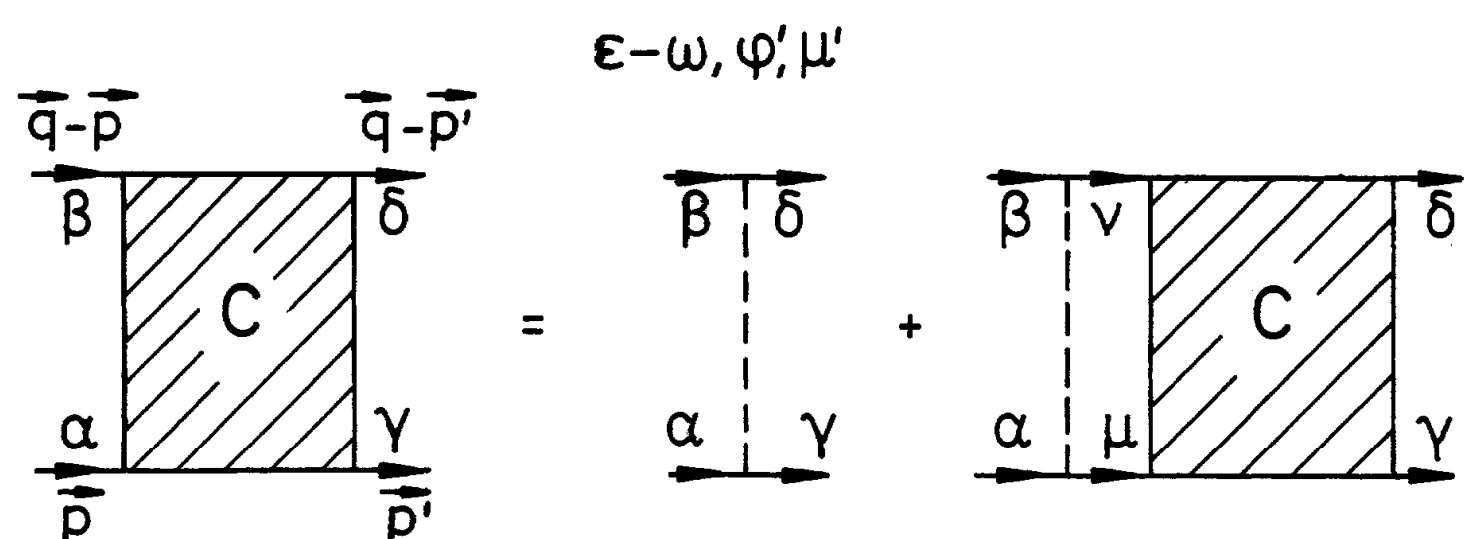

\section{$\varepsilon, \varphi, \mu$}

Fig. 4. Graphical representation of the equation determining the cooperon. There should be no problem to distinguish between the chemical potential and the spin index.

(compare Figs 4 and 5), are

$X_{\alpha \beta \gamma \delta}=X_{\alpha \beta \gamma \delta}^{0}+X_{\alpha \beta \mu \nu}^{0} \Pi_{\mu \nu}^{X} X_{\mu \nu \psi \delta}$

for $X=C, D$ (summation over $\mu, v$ implied here). The bare expressions, $X^{0}$, are given by

$2 \pi \mathscr{N}^{0} X_{\alpha \beta \gamma \delta}^{0}=\frac{1}{\tau_{0}} \delta_{\alpha \beta} \delta_{y \delta}+\frac{1}{3}\left\{\begin{array}{l}\left(\frac{1}{\tau_{s}}-\frac{1}{\tau_{s o}}\right) \boldsymbol{\sigma}_{\alpha \gamma} \cdot \boldsymbol{\sigma}_{\beta \delta} \\ \left(\frac{1}{\tau_{s}}+\frac{1}{\tau_{s o}}\right) \boldsymbol{\sigma}_{\alpha \gamma} \cdot \boldsymbol{\sigma}_{\delta \beta}\end{array}\right.$

for the cooperon and diffuson, respectively. Furthermore, for $\mu, v= \pm 1$,

$\Pi_{\mu v}^{C, D} / 2 \pi \mathscr{N}^{0} \tau=1-\tau\left(|\omega|+D q_{ \pm}^{2}+i \frac{\mu \omega_{s}-v \omega_{s}^{\prime}}{2} \operatorname{sgn} \omega\right)$

To simplify the writing, I omit in (16) and below $i \delta \mu$ sgn $\omega+\gamma_{ \pm}$, which has to be added to $|\omega|$, where the phase breaking rates $\gamma_{ \pm}$arise due to the magnetic field penetrating into the ring, $\gamma_{ \pm} \sim e^{2}\left(H \pm H^{\prime}\right)^{2} L_{\perp}^{2} D$.

For a concise summary of the results, define

$N_{1}=|\omega|+D q_{+}^{2}+2 / 3 \tau_{s}+4 / 3 \tau_{s o} ;$

$N_{2}=|\omega|+D q_{+}^{2}+2 / \tau_{s}$

and

$M_{1}=|\omega|+D q_{-}^{2}+4 / 3 \tau_{s}+4 / 3 \tau_{s o} ; \quad M_{2}=|\omega|+D q_{-}^{2}$
Then one finds

$2 \pi \mathscr{N}^{0} \tau^{2} C_{+++}=\left[N_{1}+i \operatorname{sgn} \omega\left(\omega_{s}-\omega_{s}^{\prime}\right) / 2\right]^{-1}$

$2 \pi \mathscr{N}^{0} \tau^{2} C_{+-+-}=\left[N_{1}+N_{2}-i \operatorname{sgn} \omega\left(\omega_{s}+\omega_{s}^{\prime}\right)\right] / 2 \mathscr{D}_{+}$(20)

$2 \pi \mathscr{N}^{0} \tau^{2} C_{+--+}=\left(N_{2}-N_{1}\right) / 2 \mathscr{D}_{+}$

where $\mathscr{D}_{+}=N_{1} N_{2}+\left(\omega_{s}+\omega_{s}^{\prime}\right)^{2} / 4$, and

$2 \pi \mathscr{N}^{0} \tau^{2} D_{+-+-}=\left[M_{1}+i \operatorname{sgn} \omega\left(\omega_{s}+\omega_{s}^{\prime}\right) / 2\right]^{-1}$

$2 \pi \mathscr{N}^{0} \tau^{2} D_{+++}=\left[M_{1}+M_{2}-i \operatorname{sgn} \omega\left(\omega_{s}-\omega_{s}^{\prime}\right)\right] / 2 \mathscr{D}_{-}$

$2 \pi \mathscr{N}^{0} \tau^{2} D_{++-}=\left(M_{1}-M_{2}\right) / 2 \mathscr{D}_{-}$

where $\mathscr{D}_{-}=M_{1} M_{2}+\left(\omega_{s}-\omega_{s}^{\prime}\right)^{2} / 4$. Furthermore, $C_{-+-+}=$ $C_{+-+-}^{*}, \quad C_{-++-}=C_{+-++}, C_{---}=C_{++++}^{*}, \quad$ and $D_{-+-+}=D_{+-+-}^{*}, \quad D_{--++}=D_{++--}, \quad D_{---}=$ $D_{++++}^{*}$, where the complex conjugation only operates on the $i$ explicit in (19)-(24); the other elements of $C$ and $D$ vanish. Note e.g. that the combination $\sum_{\alpha \beta} D_{\alpha \alpha \beta \beta}$ for $\omega_{s}=\omega_{s}^{\prime}$ and $\delta \mu=0$, which after analytic continuation to real frequencies enters the calculation of the classical density response, is of course unaffected by spin-flip and spin-orbit scattering, as required by particle number conservation.

\section{Correlation functions}

In the next step, it is straightforward to determine how (18) spin-effects modify the correlation functions discussed in

\section{$\overrightarrow{\mathrm{p}}-\overrightarrow{\mathrm{q}} \quad \overrightarrow{\mathrm{p}^{\prime}-\overrightarrow{\mathrm{q}}} \quad \varepsilon-\omega, \varphi^{\prime}, \mu^{\prime}$}
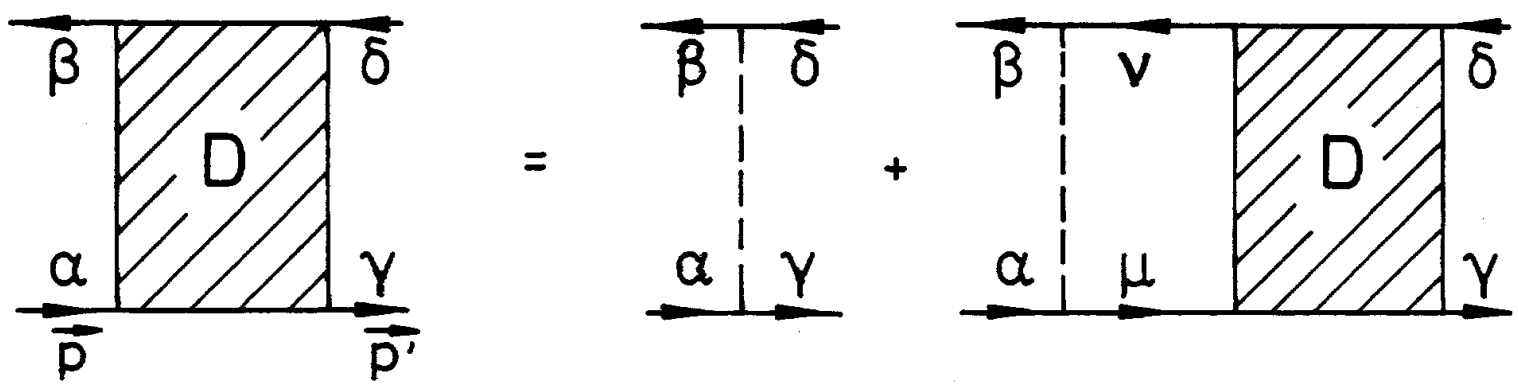

\footnotetext{
$\varepsilon, \varphi, \mu$
}

Fig. 5. Graphical representation of the equation determining the diffuson. 
Section 2 (and others). For example, the following combinations are required (see Figs 2 and 3) for $\mathscr{M}_{N}$ and $\mathscr{M}_{n}$, respectively:

$\mathscr{M}_{N}: \sum_{X=C, D} \sum_{\alpha \beta \gamma \delta} X_{\alpha \beta \gamma \delta} X_{\gamma \delta \alpha \beta}$

$\mathscr{M}_{n}: \sum_{X=C, D} \sum_{\alpha \beta} X_{\alpha \beta \alpha \beta}$

On the other hand, the potential correlator is determined by the eigenvalues, $\lambda_{j}^{X}$, of $\left\langle\alpha \beta\left|X^{0} \Pi\right| \gamma \delta\right\rangle=X_{\alpha \beta \gamma \delta}^{0} \Pi_{\gamma \delta}$, considered as $4 \times 4$-matrices, respectively:

$\mathscr{M}_{\Omega}: \sum_{X=C, D} \sum_{j=1}^{4} \ln \left(1-\lambda_{j}^{X}\right)$

Of course, (25) and (27) are consistent, as discussed in Section 2 . Here I only give the following explicit result:

$$
\begin{aligned}
\mathscr{M}_{\Omega}= & -T \sum_{\omega, q} \frac{|\omega|}{2 \pi} \ln \left\{\left[N_{1}^{2}+\frac{\left(\omega_{s}-\omega_{s}^{\prime}\right)^{2}}{4}\right]\right. \\
& \times\left[N_{1} N_{2}+\frac{\left(\omega_{s}+\omega_{s}^{\prime}\right)^{2}}{4}\right] \\
& \times\left[M_{1}^{2}+\frac{\left(\omega_{s}+\omega_{s}^{\prime}\right)^{2}}{4}\right] \\
& \left.\times\left[M_{1} M_{2}+\frac{\left(\omega_{s}-\omega_{s}^{\prime}\right)^{2}}{4}\right]\right\}
\end{aligned}
$$

In the absence of spin-effects, $N_{1}=N_{2}=|\omega|+D q_{+}^{2}$, $M_{1}=M_{2}=|\omega|+D q_{-}^{2}$, such that (6) is recovered; recall also the remark below (16).

\section{Discussion}

Without going into further detail, I discuss some general aspects of the above results. For example, it follows immediately that in the absence of spin-flip scattering, $\mathscr{M}_{\Omega}\left(\phi, \phi^{\prime}\right)=\mathscr{M}_{\Omega}\left(-\phi, \phi^{\prime}\right)$, etc., which is consistent with the expectation that the persistent current is an odd function of the flux. However, this symmetry is broken by magnetic impurities, which are assumed to have (random positions and) fixed spin directions in a given sample (thereby excluding e.g. the Kondo effect). In fact, making the impurity spins explicit, time reversal invariance implies $\Omega\left(\phi,\left\{\boldsymbol{S}_{j}\right\}\right)=\Omega(-\phi$, $\left\{-\boldsymbol{S}_{j}\right\}$ ).

Of course, the above $\mathscr{M}_{\Omega}$ has precisely the properties which follow from this general result; I note in passing that changing $\left\{\boldsymbol{S}_{j}\right\} \rightarrow\left\{-\boldsymbol{S}_{j}\right\}$ in one ring changes the sign of $1 / \tau_{s}$ in (15), and hence transforms the cooperon into the diffuson contribution, and vice versa. I emphasize that in the presence of spin scattering, the persistent current has odd and even parts (see related discussions $[30,32]$ ).

Distinguishing further between $\phi$, the Aharonov-Bohm flux entering through $q_{ \pm}$[compare (7)], and the magnetic field dependence from Zeeman splitting and field penetration, I conclude that the persistent current of a given ring has the representation

$I=\sum_{m=1}^{\infty}\left[I_{m}(H) \sin \left(2 \pi m \phi / \phi_{0}\right)+\tilde{I}_{m}(H) \cos \left(2 \pi m \phi / \phi_{0}\right]\right.$

In the absence of spin scattering, $I_{m}(H)$ and $\tilde{I}_{m}(H)$ are even and odd functions, respectively, while in the presence of spin scattering, they also acquire odd and even contributions. In particular, for strong spin-flip scattering, it follows that the cooperon is suppressed entirely, while the diffuson part is reduced by four [28] compared to its ideal value [compare (8) and (10)]. Thus, in this limit, I conclude that $\left\langle\tilde{I}_{m}^{2}\right\rangle_{c}=$ $\left\langle I_{m}^{2}\right\rangle_{c}$, and that these are $\frac{1}{4}$ of the ideal value (10).

$I$ remark that, for the average current, $\mathscr{M}_{N}$ is required for equal fluxes [see (4)], i.e. only the cooperon contribution, which is strongly reduced for strong spin-flip scattering. In any case, the average current is odd

Finally, interaction induced persistent currents have been discussed in considerable detail $[7,8,11,17,18,21]$. Here I remark only that a rough estimate can be obtained by considering the Hartree contribution to the grand potential,

$\Omega_{H}=\frac{1}{2} \int d \boldsymbol{r} \int d \boldsymbol{r}^{\prime} \tilde{u}\left(\boldsymbol{r}-\boldsymbol{r}^{\prime}\right) \delta n(\boldsymbol{r}) \delta n\left(\boldsymbol{r}^{\prime}\right)$

where $\tilde{u}$ denotes the screened Coulomb potential, and the estimate below (13) for the local correlations. This leads immediately to the result $\left\langle\Omega_{H}\right\rangle \sim \lambda_{c} E_{c}$, where $\lambda_{c} \sim$ $\mathcal{N}^{0} \tilde{u}\left(k \sim k_{F}\right)$ is the dimensionless coupling constant. More detailed investigations show, however, that $\lambda_{c}$ is reduced logarithmically below its bare value $[8,24,25,29]$, and that it is essential to include the exchange contribution when studying the spin dependence: For example, the remarkable result that the average interaction induced persistent current is essentially independent of spin-orbit scattering (except for minor changes in the reduction factor), arises from a subtle interplay between direct and exchange terms [8].

Applying (30) also naively to the fluctuations [17], one realizes that since $\mathscr{M}_{n}$ consists additively of a regular and a flux sensitive ("singular") contribution, the dominant flux sensitive part contains the product of these, which led [17] to $\mathscr{M}_{\Omega}^{\mathrm{int}} \sim E_{c} / \tau$, which is larger by a factor $\sim(L / l)^{2}$ than the non-interacting electron result (10). Subsequent analysis showed, however, that the arguments are much more subtle $[18,21]$. In particular, the most recent effort [21] contains an attempt at a systematic Hartree-type theory, excluding the exchange terms; in view of what was remarked above concerning the spin dependence of the average interaction contribution, the spin dependence of $\mathscr{M}_{\Omega}^{\text {int }}$ is an open problem.

\section{Acknowledgements}

I wish to thank J. Kroha, J. Rammer, A. Schmid, and A. L. Shelankov for discussions.

\section{References}

1. Büttiker, M., Imry, Y. and Landauer, R., Phys. Lett. 96A, 365 (1985).

2. Cheung, H.-F., Gefen, Y. and Riedel, E. K., IBM J. Res. Develop. 32, 359 (1988).

3. Entin-Wohlmann, O. and Gefen, Y., Europhys. Lett. 8, 477 (1989).

4. Cheung, H.-F., Riedel, E. K. and Gefen, Y., Phys. Rev. Lett. 62, 587 (1989).

5. Bouchiat, H. and Montambaux, G., J. Phys. (Paris) 50, 2695 (1989).

6. Montambaux, G., Bouchiat, H., Sigeti, D. and Friesner, R., Phys. Rev. B42, 7647 (1990).

7. Ambegaokar, V. and Eckern, U., Phys. Rev. Lett. 65, 381 (1990); 67, 3192 (1991).

8. Eckern, U., Z. Phys. B82, 393 (1991).

9. Imry, Y., "Quantum Coherence in Mesoscopic Systems" (Edited by B. Kramer) (Plenum Press, New York 1991), NATO ASI, Series B: Physics, vol. 254, p. 221. 
10. Akkermans, E., Auerbach, A., Avron, J. E. and Shapiro, B., Phys. Rev. Lett. 66, 76 (1991).

11. Schmid, A., Phys. Rev. Lett. 66, 80 (1991)

12. von Oppen, F. and Riedel, E. K., Phys. Rev. Lett. 66, 84 (1991).

13. Altshuler, B. L., Gefen, Y. and Imry, Y., Phys. Rev. Lett. 66, 88 (1991).

14. Efetov, K. B., Phys. Rev. Lett. 66, 2794 (1991).

15. Riedel, E. K. and von Oppen, F., preprint (May 1991).

16. Mathur, H. and Stone, A. D., Phys. Rev. B44, 10957 (1991).

17. Eckern, U. and Schmid, A., Europhys. Lett. 18, 457 (1992).

18. Smith, R. A. and Ambegaokar, V., Europhys. Lett. 20, 161 (1992).

19. Altland, A., Iida, S., Müller-Groeling, A. and Weidenmüller, H. A., Europhys. Lett. 20, 155 (1992).

20. Szafer, A. and Altshuler, B. L., Phys. Rev. Lett. 70, 587 (1993).

21. Eckern, U. and Schmid, A., Ann. Physik (Leipzig), 2, 180 (1993).

22. Levy, L. P., Dolan, G., Dunsmuir, J. and Bouchiat, H., Phys. Rev. Lett. 64, 2074 (1990); Levy, L. P., Physica B169, 245 (1991).

23. Chandrasekhar, V., Webb, R. A., Brady, M. J., Ketchen, M. B., Gallagher, W. J. and Kleinsasser, A., Phys. Rev. Lett. 67, 3578 (1991).
24. Altshuler, B. L., Aronov, A. G., Khmelnitskii, D. E. and Larkin, A. I., "Quantum Theory of Solids" (Edited by I. M. Lifshitz) (MIR Publishers, Moscow 1980), p. 130.

25. Altshuler, B. L. and Aronov, A. G., "Electron-Electron Interactions in Disordered Systems" (Edited by. A. L. Efros and M. Pollak) (NorthHolland, Amsterdam 1985), p. 1.

26. Hikami, S., Larkin, A. I. and Nagaoka, Y., Progr. Theor. Phys. 63, 707 (1980).

27. Maekawa, S. and Fukuyama, H., J. Phys. Soc. Japan 50, 2516 (1981).

28. Altshuler, B. L. and Shklovskii, B. I., Sov. Phys. JETP 64, 127 (1986).

29. Altshuler, B. L., Aronov, A. G. and Zyuzin, A. Yu., Sov. Phys. JETP 57, 889 (1983).

30. Altshuler, B. L. and Spivak, B. Z., JETP Lett. 42, 447 (1985).

31. The notation here differs from Ref. [8]: $C_{\alpha \gamma \beta \delta} \equiv K_{\alpha \gamma \beta \delta}$.

32. Büttiker, M., Phys. Rev. Lett. 57, 1761 (1986) 